\title{
Toric matrix Schubert varieties and root polytopes (extended abstract)
}

\author{
Laura Escobar杵 and Karola Mészáros"非 \\ ${ }^{1}$ Department of Mathematics, University of Illinois at Urbana-Champaigh, Urbana, IL 61801, USA \\ ${ }^{2}$ Department of Mathematics, Cornell University, Ithaca, NY 14853, USA
}

\begin{abstract}
Start with a permutation matrix $\pi$ and consider all matrices that can be obtained from $\pi$ by taking downward row operations and rightward column operations; the closure of this set gives the matrix Schubert variety $\overline{X_{\pi}}$. We characterize when the ideal defining $\overline{X_{\pi}}$ is toric (with respect to a $2 n-1$-dimensional torus) and study the associated polytope of its projectivization. We construct regular triangulations of these polytopes which we show are geometric realizations of a family of subword complexes. We also show that these complexes can be realized geometrically via regular triangulations of root polytopes. This implies that a family of $\beta$-Grothendieck polynomials are special cases of reduced forms in the subdivision algebra of root polytopes. We also write the volume and Ehrhart series of root polytopes in terms of $\beta$-Grothendieck polynomials. Subword complexes were introduced by Knutson and Miller in 2004, who showed that they are homeomorphic to balls or spheres and raised the question of their polytopal realizations.

Résumé. En partant d'une matrice de permutation $\pi$, considérons toutes les matrices qui peuvent être obtenues à partir de $\pi$ en effectuant des opérations de ligne vers le bas et des opérations de colonne vers la droite ; l'adhérence de cet ensemble donne la variété Schubert de matrices $\overline{X_{\pi}}$. Nous caractérisons la situation où l'idéal définissant $\overline{X_{\pi}}$ est torique et étudions le polytope associé de sa projectivisation. Nous construisons des triangulations régulières de ces polytopes et nous montrons qu'elles sont des réalisations géométriques d'une famille de complexes de sous-mots. Nous montrons également que ces complexes peuvent être réalisés géométriquement par des triangulations régulières de polytopes de racines. Cela implique qu'une famille de polynômes $\beta$-Grothendieck sont des cas particuliers de formes réduites dans l'algèbre de subdivision de polytopes des racines. On peut aussi écrire le volume et la série d'Ehrhart des polytopes des racines en termes de $\beta$-Grothendieck polynômes. Les complexes de sous-mots ont été introduits par Knutson et Miller en 2004, qui ont soulevé la question de leurs réalisations polytopales.
\end{abstract}

Keywords. subword complex, root polytope, matrix Schubert variety, toric variety

\section{Introduction}

This is an extended abstract based on Escobar and Mészáros (2015a) and Escobar and Mészáros (2015b). We study the geometry of matrix Schubert varieties and give geometric realizations of a family of subword complexes. Matrix Schubert varieties were introduced by Fulton (1992) to study the degeneraci loci of

\footnotetext{
†Email: lescobar@illinois.edu.

‡Mészáros was partially supported by a National Science Foundation Grant (DMS 1501059).
} 
flagged vector bundles. Knutson and Miller (2005) showed that Schubert polynomials are multidegrees of matrix Schubert varieties. Knutson and Miller (2004, 2005) introduced subword complexes to illustrate the combinatorics of Schubert polynomials and determinantal ideals, building up on the work of Fomin and Kirillov (1994); Bergeron and Billey (1993). Knutson and Miller proved that any subword complex is homeomorphic to a ball or a sphere and asked about their geometric realizations.

Given a matrix Schubert variety $\overline{X_{\pi}}$, it can be written as $\overline{X_{\pi}}=Y_{\pi} \times \mathbb{C}^{q}$ (where $q$ is maximal possible). Our main results are as follows. We characterize when $Y_{\pi}$ is toric (with respect to a $\left(\mathbb{C}^{*}\right)^{2 n-1}$-action) and study the polytope $\Phi\left(\mathbb{P}\left(Y_{\pi}\right)\right)$ corresponding to its projectivization. We construct a regular triangulation of $\Phi\left(\mathbb{P}\left(Y_{\pi}\right)\right)$, induced from a degeneration to a root polytope, which we show are geometric realizations of a family of subword complexes. The following papers have partially answered the question about the geometric realization of spherical subword complexes: Stump (2011); Ceballos (2012); Pilaud and Pocchiola (2012); Pilaud and Santos (2012); Serrano and Stump (2012); Ceballos et al. (2014); Bergeron et al. (2015). This submission is based on Escobar and Mészáros (2015a b), where we give the first realizations of a family of subword complexes which are homeomorphic to balls.

The roadmap of this paper is as follows. In Section 2 we define matrix Schubert varieties $\overline{X_{\pi}}$ and calculate the moment polytope $\Phi\left(\mathbb{P}\left(Y_{\pi}\right)\right)$ of the projectivization of $Y_{\pi}$. In Section 3 we characterize when $Y_{\pi}$ is toric and construct a regular triangulation of $\Phi\left(\mathbb{P}\left(Y_{\pi}\right)\right)$. In Section 4 we define subword complexes, give geometric realizations of subword complexes homeomorphic to balls, and show how to express the volume and Ehrhart series of root polytopes in terms of Grothendieck polynomials. Finally, in Section 5 we give canonical triangulations of $\Phi\left(\mathbb{P}\left(Y_{\pi}\right)\right)$ and show they are geometric realizations of pipe dream complexes for all $\pi$ such that $Y_{\pi}$ is toric.

\section{Matrix Schubert varieties}

Given a matrix Schubert variety $\overline{X_{\pi}}$ we define a variety $Y_{\pi} \hookrightarrow \overline{X_{\pi}}$ and characterize for which $\pi$, the variety $Y_{\pi}$ is toric using the diagram of $\pi$. For such $\pi$, we construct a regular triangulation of its corresponding polytope, which we show is a geometric realization of a family of subword complexes, see Proposition 3.1 and Theorem 5.3 .

Let $M_{n}$ denote $n \times n$ matrices over $\mathbb{C}, B_{+}$denote upper triangular invertible $n \times n$ matrices and $B_{-}$ denote lower triangular invertible $n \times n$ matrices. We let $\pi \in S_{n}$ denote both a permutation and its corresponding permutation matrix, where its $(i, j)$-th entry is

$$
(\pi)_{(i, j)}=\left\{\begin{array}{l}
1, \text { if } \pi(j)=i, \\
0, \text { else }
\end{array}\right.
$$

The multiplication on the left by matrices in $B_{-}$corresponds to downward row operations and multiplication on the right by matrices in $B_{+}$corresponds to rightward column operations. This multiplication gives a left action of $B_{-} \times B_{+}$on $M_{n}$ defined by

$$
(X, Y) \cdot M:=X M Y^{-1} .
$$

Given $1 \leq a \leq m$ and $1 \leq b \leq m$, let $M_{(a, b)}$ denote the upper left $a \times b$ submatrix of the matrix $M$. Define a rank function of a matrix $M$ to be $r_{M}(a, b):=\operatorname{rank}\left(M_{(a, b)}\right)$. We then have that $M \in B_{-} \pi B_{+}$ if and only if $r_{M}(a, b)=r_{\pi}(a, b)$ for all $(a, b) \in[m] \times[m]$. 
Definition 2.1 The matrix Schubert variety of $\pi$ is $\overline{X_{\pi}}:=\overline{B_{-} \pi B_{+}}$, i.e. the Zariksi closure of its $\left(B_{-} \times B_{+}\right)$-orbit inside $M_{n}=\mathbb{C}^{n^{2}}$.

Fulton studied this affine variety in Fulton (1992). We summarize some of his results here.

Theorem 2.2 (Fulton 1992 Proposition 3.3) The matrix Schubert variety $\overline{X_{\pi}}$ is an irreducible variety of dimension $n^{2}-\ell(\pi)$ defined as a scheme by the equations $r_{M}(a, b) \leq r_{\pi}(a, b)$ for all $(a, b) \in[n] \times[n]$.

Some of these inequalities are implied by others, and Fulton described the minimal set of rank conditions.

Definition 2.3 The (Rothe) diagram of a permutation $\pi$ is the collection of boxes $D(\pi)=\left\{\left(\pi_{j}, i\right): i<\right.$ $\left.j, \pi_{i}>\pi_{j}\right\}$. It can be visualized by considering the boxes left in the $n \times n$ grid after we cross out the boxes appearing south and east of each 1 in the permutation matrix for $\pi$.

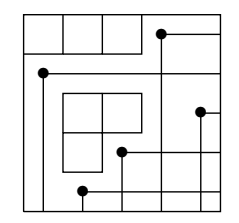

Fig. 1: The diagram for $\pi=[25413]$.

Definition 2.4 Fulton's essential set $E s s(\pi)$ is the set consisting of the south-east corners of $D(\pi)$.

Theorem 2.5 (Fulton 1992 Lemma 3.10) The ideal defining the variety $\bar{X}_{\pi}$ is generated by the equations $r_{M}(a, b) \leq r_{\pi}(a, b)$ for all $(a, b) \in \operatorname{Ess}(\pi)$.

We now define some regions inside the $(n \times n)$-grid and some varieties corresponding to these regions, including $Y_{\pi}$.

Definition 2.6 The dominant piece, denoted dom $(\pi)$, of a permutation $\pi$ is the connected component of the diagram of $\pi$ containing the box $(1,1)$, or empty if $\pi(1)=1$.

Definition 2.7 Let $\boldsymbol{N W}(\boldsymbol{\pi})$ denote the union over the entries north-west of some box in $D(\pi)$. Let $\boldsymbol{L}(\boldsymbol{\pi}):=N W(\pi)-\operatorname{dom}(\pi)$ and let $\boldsymbol{L}^{\prime}(\boldsymbol{\pi}):=L(\pi)-D(\pi)$.

See Figure 2 for an example.

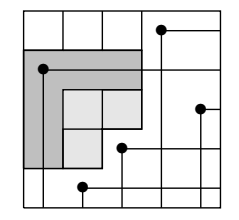

Fig. 2: Given $\pi=[25413], L(\pi)$ consists of all the gray boxes and $L^{\prime}(\pi)$ consists of only the darker gray boxes.

Definition 2.8 Given a permutation $\pi$, let $\boldsymbol{Y}_{\boldsymbol{\pi}}$ be the projection of $\overline{X_{\pi}}$ onto the entries inside $L(\pi)$ and let $\boldsymbol{V}_{\boldsymbol{\pi}}$ be the projection onto the entries not north-west of any box of $D(\pi)$. 
Theorem 2.5 implies that the entries in $V_{\pi}$ are free in $\overline{X_{\pi}}$ and thus $V_{\pi} \cong \mathbb{C}^{q}$, where $q$ is the number of boxes in the region defining $V_{\pi}$, and that $\bar{X}_{\pi}=Y_{\pi} \times V_{\pi}$. This, together with Theorem 2.2 imply that $\operatorname{dim}\left(Y_{\pi}\right)=\left|L^{\prime}(\pi)\right|$ and that $Y_{\pi}$ is irreducible.

Let $T^{n}$ consist of $n \times n$ diagonal invertible matrices. The action defined in Equation (1) restricts to a $\left(T^{n} \times T^{n}\right)$-action on $M_{n}$. This yields a $\left(T^{n} \times T^{n}\right)$-action on $\overline{X_{\pi}}$ with $\operatorname{Stab}\left(T^{2 n}\right)=\left\{(a \cdot I, a \cdot I): a \in \mathbb{C}^{*}\right\}$, as well as an action on $Y_{\pi}$ and $V_{\pi}$. In Theorem 3.3 we characterize the $\pi$ for which $Y_{\pi}$ is a toric variety with respect to $T^{2 n} / \operatorname{Stab}\left(T^{2 n}\right)$ in terms of the shape of $L^{\prime}(\pi)$. In other words, we characterize the $\pi$ such that $Y_{\pi}$ has a dense $T^{2 n}$-orbit. We denote the quotient $T^{2 n} / \operatorname{Stab}\left(T^{2 n}\right)$ by $T^{2 n-1}$. Note that $\overline{X_{\pi}}$ and $Y_{\pi}$ are normal varieties by Fulton's realizations in Fulton (1992) as subvarieties of Schubert varieties, which are normal by De Concini and Lakshmibai (1981); Ramanan and Ramanathan (1985).

Since $Y_{\pi}$ is an irreducible variety and toric varieties are also irreducible, in order to show that $Y_{\pi}$ is a toric variety with respect to $T^{2 n-1}$, it suffices to show that it has the same dimension as some $T^{2 n-1}$ orbit. When $p$ is a general point of $Y_{\pi}$, then $\overline{T^{2 n} \cdot p} \subset Y_{\pi}$ is the affine toric variety associated to the $T^{2 n}$-momen ${ }^{\text {(i) }}$ cone of $Y_{\pi}$, which we denote by $\boldsymbol{\Phi}\left(\boldsymbol{Y}_{\pi}\right)$, and $\operatorname{dim}\left(\overline{T^{2 n} \cdot p}\right)=\operatorname{dim}\left(\Phi\left(Y_{\pi}\right)\right)$. In Theorem 3.3 we classify when $Y_{\pi}$ is a toric variety by classifying the $\pi$ for which $\operatorname{dim}\left(\Phi\left(Y_{\pi}\right)\right)=\operatorname{dim}\left(Y_{\pi}\right)$.

To compute the dimension of the cone $\Phi\left(Y_{\pi}\right)$, we start by describing the cone $\Phi\left(\overline{X_{\pi}}\right)$ corresponding to a $T^{2 n}$-orbit of a general point $q$ in $\overline{X_{\pi}}$; without loss of generality $q=(1, \ldots, 1)$. The orbit $\overline{T^{2 n} \cdot q}$ is the Zariski closure of the image of a map $\varphi: T^{2 n} \rightarrow \mathbb{C}^{n^{2}}$ where $\varphi(t)=\left(t^{a_{(1,1)}} q_{(1,1)}, \ldots, t^{a_{(n, n)}} q_{(n, n)}\right)$ and $\Phi\left(\overline{X_{\pi}}\right)$ is the cone spanned by the exponents $a_{(i, j)}$ of the monomials. Notice that the exponents are $x_{i}-y_{j}$, where the $x_{i}$ are the standard basis for $\mathbb{R}^{n} \times 0$, and the $y_{j}$ are the standard basis for $0 \times \mathbb{R}^{n}$, because if $A$ and $B$ are the diagonal matrices with diagonal entries $\left(a_{1}, \ldots, a_{n}\right)$ and $\left(b_{1}, \ldots, b_{n}\right)$, respectively, then for any matrix $M$ the $(i, j)$-th entry of $A M B^{-1}$ is $a_{i} b_{j}^{-1} M_{(i, j)}$. It follows that the moment cone $\Phi\left(\overline{X_{\pi}}\right)$ is the cone spanned by the vectors in the set $\left\{x_{i}-y_{j} \mid(i, j) \in[n] \times[n]\right\}$. Now $Y_{\pi} \hookrightarrow \overline{X_{\pi}}$ by restricting $\overline{X_{\pi}}$ to the entries inside $L(\pi)$, and so $\Phi\left(Y_{\pi}\right)$ is the cone spanned by the set $\left\{x_{i}-y_{j} \mid(i, j) \in L(\pi)\right\}$.

The variety $\overline{X_{\pi}}$ is a cone, meaning that for any $z \in \overline{X_{\pi}}$ and $c \in \mathbb{C}$, we have that $c z \in \overline{X_{\pi}}$. We can therefore projectivize it, that is, we can take the projective variety

$$
\mathbb{P}\left(\overline{X_{\pi}}\right):=\left\{\left[z_{(1,1)}, \ldots, z_{(n, n)}\right]:\left(z_{(1,1)}, \ldots, z_{(n, n)}\right) \in \overline{X_{\pi}}\right\} \subset \mathbb{C P}^{n^{2}-1},
$$

and the same is true for $Y_{\pi}$. In this paper we study the moment (ii) $_{\text {polytope }} \Phi\left(\mathbb{P}\left(\boldsymbol{Y}_{\pi}\right)\right)$ of the projectivization of $Y_{\pi}$. This polytope is the convex hull of $\left(x_{i}-y_{j}\right)$ for $(i, j)$ inside $L(\pi)$. The next section studies the properties of the moment polytopes $\Phi\left(\mathbb{P}\left(Y_{\pi}\right)\right)$.

\section{Understanding the polytope $\Phi\left(\mathbb{P}\left(Y_{\pi}\right)\right)$}

In this section we describe the polytope $\Phi\left(\mathbb{P}\left(Y_{\pi}\right)\right)=\operatorname{ConvHull}\left(x_{i}-y_{j} \mid(i, j) \in L(\pi)\right)$ for $\pi \in S_{n}$, the moment polytope of the projectivization of $Y_{\pi}$. This polytope is a root polytope, since its vertices are positive roots of type $A_{n-1}$. We will encounter slightly different root polytopes (acyclic root polytopes) in Section 4.2 when describing the realizations of a family of pipe dream complexes. In Section 5 we will give a map that transforms the root polytope $\Phi\left(\mathbb{P}\left(Y_{\pi}\right)\right)$ into an acyclic root polytope for $\pi=1 \pi^{\prime}$ with $\pi^{\prime}$ dominant. We set our notation for the first root polytopes now.

(i) The reason we use the word moment for these convex objects is because they arise in the context of symplectic and pre-symplectic geometry. For readers interested in the connection, we refer them to Cannas da Silva 2001, 2003); Berline and Vergne (2011).

(ii) See footnote (i) 


\subsection{Root polytopes and their triangulations}

A root polytope (of type $A_{n-1}$ ) is the convex hull of some of the points $e_{i}-e_{j}$ for $1 \leq i<j \leq n$. Given a graph $G$ on the vertex set $[n]$ we associate to it the root polytope

$$
Q_{G}=\operatorname{ConvHull}\left(e_{i}-e_{j} \mid(i, j) \in E(G), i<j\right)
$$

Note that for every $\pi \in S_{n}$ we have that $L(\pi)$ is a skew Ferrers diagram. Given a skew Ferrers diagram $D$ with $r$ rows and $c$ columns, label its rows by $1,2, \ldots, r$ from top to bottom and its columns by $1,2, \ldots, c$ from left to right. Define

$$
G_{D}=\left(\left\{x_{1}, \ldots, x_{r}, y_{1}, \ldots, y_{c}\right\},\left\{\left(x_{i}, y_{j}\right) \mid(i, j) \in D\right\}\right) .
$$

Then

$$
\Phi\left(\mathbb{P}\left(Y_{\pi}\right)\right)=Q_{G_{L(\pi)}} .
$$

Note that an edge $\left(x_{i}, y_{j}\right) \in G_{D}$ yields the vertex $e_{i}-e_{r+j}$ of the root polytope $Q_{G_{D}}$.

Given a drawing of a graph $G$ so that its vertices $v_{1}, \ldots, v_{n}$ are arranged in this order on a horizontal line, and its edges are drawn above this line, we say that $G$ is noncrossing if it has no edges $\left(v_{i}, v_{k}\right)$ and $\left(v_{j}, v_{l}\right)$ with $i<j<k<l$. A vertex $v_{i}$ of $G$ is said to be nonalternating if it has both an incoming and an outgoing edge; it is called alternating otherwise. The graph $G$ is alternating if all its vertices are alternating.

Since being noncrossing depends on the drawing of the graph it is essential that we set a way to draw $G_{D}$. For the purposes of this paper the vertices of $G_{D}$ are drawn from left to right in the following order: $x_{r}, \ldots, x_{1}, y_{c}, \ldots, y_{1}$.

Lemma 3.1 Given a skew diagram $D$ for which $G_{D}$ has $k$ components, the root polytope $Q_{G_{D}}=$ $\bigcup_{F} Q_{F}$, where the union runs over all noncrossing alternating spanning forests of $G_{D}$ with $\left|V\left(G_{D}\right)\right|-k$ edges and the simplices $Q_{F}$ are interior disjoint and of the same dimension as $Q_{G_{D}}$.

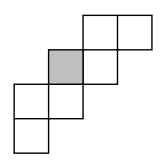

Fig. 3: For $D$ the unshaded region, $G_{D}$ is disconnected. In this case, $D^{\prime}$ equals $D$ together with the shaded square.

We call the triangulation of $Q_{G_{D}}$ given in Lemma 3.1 the noncrossing alternating triangulation, or NAT for short. These triangulations are closely related to the triangulations appearing in Gelfand et al. (1997) and Cellini and Marietti (2014). Recall that a triangulation of a polytope $P$ is regular if there exists a concave piecewise linear function $f: P \rightarrow \mathbb{R}$ such that the regions of linearity of $f$ are the maximal simplices in the triangulation.

Proposition 3.1 For a skew diagram D, the NAT triangulation of $Q_{G_{D}}$ described in Lemma 3.1 is a regular triangulation. 


\subsection{Characterizing when $Y_{\pi}$ is a toric variety}

Now we are ready to use the above lemmas in order to characterize when $Y_{\pi}$ is a toric variety.

Lemma 3.2 Given a skew diagram $D$ with $r$ rows and c columns, for which $G_{D}$ has $k$ components, the dimension of $Q_{G_{D}}$ is $r+c-k-1$.

Theorem 3.3 $Y_{\pi}$ is a toric variety with respect to the $T^{2 n-1}$-action if and only if $L^{\prime}(\pi)$ consists of disjoint hooks that do not share a row or a column with each other.

Proof: We have that $\operatorname{dim}\left(Y_{\pi}\right)=\left|L^{\prime}(\pi)\right|$. Lemma 3.2 yields that the dimension of $\Phi\left(\mathbb{P}\left(Y_{\pi}\right)\right)$ equals $\left|L^{\prime}(\pi)\right|-1$ if and only if $L^{\prime}(\pi)$ consists of disjoint hooks that do not share a row or a column with each other. This suffices to prove the theorem.

A dominant permutation is one for which its diagram has empty dominant piece and is in the shape of a partition. An immediate corollary of Theorem 3.3 is the following.

Corollary 3.4 If $\pi^{\prime}$ is a dominant permutation on $2,3, \ldots, n$ then $Y_{1 \pi^{\prime}}$ is a toric variety.

\section{On geometric realizations of subword complexes}

In this section we give some geometric realizations of subword complexes homeomorphic to balls. In Section 4.1 we show that the NAT triangulations studied in the previous section geometrically realize certain subword complexes. In Section 4.2 we use acyclic root polytopes to give geometric realizations for pipe dream complexes of permutations $\pi=1 \pi^{\prime}$ with $\pi^{\prime}$ dominant.

The symmetric group $S_{n}$ is generated by the adjacent transpositions $s_{1}, \ldots, s_{n-1}$, where $s_{i}$ transposes $i \leftrightarrow i+1$. Let $Q=\left(q_{1}, \ldots, q_{m}\right)$ be a word in $\left\{s_{1}, \ldots, s_{n-1}\right\}$. A subword $J=\left(r_{1}, \ldots, r_{m}\right)$ of $Q$ is a word obtained from $Q$ by replacing some of its letters by -. There are a total of $2^{|Q|}$ subwords of $Q$. Given a subword $J$, we denote by $Q \backslash J$ the subword with $k$-th entry equal to - if $r_{k} \neq-$ and equal to $q_{k}$ otherwise for, $k=1, \ldots, m$. For example, $J=\left(s_{1},-, s_{3},-, s_{2}\right)$ is a subword of $Q=\left(s_{1}, s_{2}, s_{3}, s_{1}, s_{2}\right)$ and $Q \backslash J=\left(-, s_{2},-, s_{1},-\right)$. Given a subword $J$ we denote by $\prod J$ the product of the letters in $J$, from left to right, with - behaving as the identity.

Definition 4.1 Knutson and Miller (2004, 2005) Let $Q=\left(q_{1}, \ldots, q_{m}\right)$ be a word in $\left\{s_{1}, \ldots, s_{n-1}\right\}$ and $\pi \in S_{n}$. The subword complex $\Delta(Q, \pi)$ is the simplicial complex on the vertex set $Q$ whose facets are the subwords $F$ of $Q$ such that the product $\Pi(Q \backslash F)$ is a reduced expression for $\pi$. The pipe dream complex $P D(\pi)$ is the subword complex $\Delta(Q, \pi)$ corresponding to the triangular word $Q=$ $\left(s_{n-1}, s_{n-2}, s_{n-1}, \ldots, s_{1}, s_{2}, \ldots, s_{n-1}\right)$ and $\pi$.

\subsection{Realization by NAT triangulations}

Given a permutation $\pi \in S_{n}$, let $\overline{L(\pi)}$ be the mirror image of the skew shape $L(\pi)$. Fill in the boxes of $\overline{L(\pi)}$ with transpositions starting with $s_{1}, s_{2}, \ldots$ on the first column, $s_{2}, s_{3}, \ldots$ on the second column, and so on. Let $Q(\overline{L(\pi)})$ be the word given by reading the transpositions in the boxes of $\overline{L(\pi)}$ from left to right and from bottom to top. Let $P(\pi)=\overline{L(\pi)}-B(\pi)$ where $B(\pi)$ is as follows. In each connected part of $\overline{L(\pi)}$ draw the lowestmost path from its top left box to its bottommost rightmost box. These boxes constitute $B(\pi)$. Let $p(\pi)$ be the permutation obtained from reading the transpositions in the boxes of $P(\pi)$ from left to right and from bottom to top. See Figure 4 

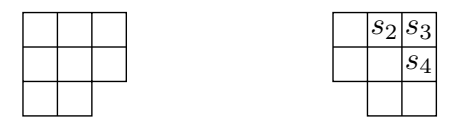

Fig. 4: On the left we have $L(\pi)$ and on the right $\overline{L(\pi)}$ for $\pi=[14325]$. Note that $p(\pi)=s_{4} s_{2} s_{3}=[13524]$.

Theorem 4.2 For $\pi \in S_{n}$, the noncrossing alternating triangulation of the root polytope $Q_{G_{L(\pi)}}$ is a geometric realization of the subword complex $\Delta(Q(\overline{L(\pi)}), p(\pi))$.

\subsection{Realizations for pipe dream complexes of dominant permutations by acyclic root polytopes}

In this section we give a geometric realization for a different family of subword complexes using acyclic root polytopes. We show that the pipe dream complex $P D(\pi)$ of a permutation $\pi=1 \pi^{\prime}$, with $\pi^{\prime}$ dominant, can be geometrically realized as the canonical triangulation of an acyclic root polytope $\mathcal{P}(T(\pi))$. These polytopes are closely related to the root polytopes of Section 3.1

We begin by defining acyclic root polytopes. Let $G$ be an acyclic graph on the vertex set $[n+1]$. Define

$$
\begin{gathered}
\mathcal{V}_{G}=\left\{e_{i}-e_{j} \mid(i, j) \in E(G), i<j\right\}, \text { a set of vectors associated to } G ; \\
\operatorname{cone}(G)=\left\langle\mathcal{V}_{G}\right\rangle:=\left\{\sum_{e_{i}-e_{j} \in \mathcal{V}_{G}} c_{i j}\left(e_{i}-e_{j}\right) \mid c_{i j} \geq 0\right\}, \text { the cone associated to } G \text {; and } \\
\overline{\mathcal{V}}_{G}=\Phi^{+} \cap \operatorname{cone}(G), \text { all the positive roots of type } A_{n} \text { contained in cone }(G),
\end{gathered}
$$

where $\Phi^{+}=\left\{e_{i}-e_{j} \mid 1 \leq i<j \leq n+1\right\}$ is the set of positive roots of type $A_{n}$. The acyclic root polytope $\mathcal{P}(G)$ associated to the acyclic graph $G$ is

$$
\mathcal{P}(G)=\operatorname{ConvHull}\left(0, e_{i}-e_{j} \mid e_{i}-e_{j} \in \overline{\mathcal{V}}_{G}\right)
$$

Theorem 4.3 Mészáros (2011) Let $T_{1}, \ldots, T_{k}$ be the noncrossing alternating spanning trees of the directed transitive closure of the acyclic graph $G$. Then $\mathcal{P}\left(T_{1}\right), \ldots, \mathcal{P}\left(T_{k}\right)$ are top dimensional simplices in a regular triangulation of $\mathcal{P}(G)$ called the canonical triangulation.

The main tool developed in Mészáros (2011) which is used to construct the canonical triangulation of Theorem 4.3 is the subdivision algebra. Subdivision algebras have since been utilized in solving various problems in Mészáros (2014); Mészáros (2015); Mészáros (2015); Mészáros (2016); Mészáros (2015); Mészáros and Morales (2015).

When $P D(\pi)$ is not a ball, it is usually a cone over a list of its vertices, namely those that are in all its facets. Let cone $(\pi)$ denote the set of vertices of $P D(\pi)$ that are in all its facets. We define the core of $\pi$ to be the restriction of $P D(\pi)$ to the set of vertices not in cone $(\pi)$. Then $P D(\pi)$ is obtained from its core by iteratively coning core $(\pi)$ over the vertices in cone $(\pi)$. Translating to pipe dream complexes, the core is the restriction to the entries in the $n \times n$ matrix that are a cross in some reduced pipe dream for $\pi$. We refer to the region itself as the core region, and denote it by $\operatorname{cr}(\pi)$. Let $\pi=1 \pi^{\prime}$, where $\pi^{\prime}$ is dominant. Denote by $\mathcal{S}(\boldsymbol{\pi})$ the subword complex which is the core $(\pi)$ coned over the vertex of $P D(\pi)$ corresponding to the entry $(1,1)$. Denote the region which is the union of $(1,1)$ and $\operatorname{cr}(\pi)$ by $\boldsymbol{R}(\boldsymbol{\pi})$. 
Proposition 4.1 Let $\pi=1 \pi^{\prime}$ with $\pi^{\prime}$ dominant. Then $R(\pi)=N W(\pi)-E s s(\pi)$.

The SE boundary of the core region starting from the southwest (SW) corner of it to the northeast (NE) corner can be described as a series of east (E) and north (N) steps. We construct the graph $T(\pi)$ by looking at the $E$ and $N$ steps bounding the $S E$ boundary of $N W(\pi)-E s s(\pi)$. Let $A$ be the set consisting of all the $N$ steps together with the $E$ steps that do not bound a box in $\operatorname{Ess}(\pi)$. Suppose $|A|=m$, as we travel the SE boundary from the $S W$ corner, we label, in order, the $E$ steps and $N$ steps in $A$ with the elements of the sequence $\left(\alpha_{1}, \ldots, \alpha_{m}\right)$. For the $E$ steps that we did not assign an $\alpha_{i}$, we consider their label to be the $\alpha_{i}$ assigned to the $N$ step directly preceding them. See Figure 6 for an example of the labelling.

Given a diagram of a permutation there are two reduced pipe dreams for $\pi$ with special names: the bottom reduced pipe dream of $\pi$ obtained by aligning the diagram to the left and replacing the boxes with crosses, and the top reduced pipe dream of $\pi$ obtained in a similar fashion, but by aligning the diagram up. See Figure 5 for an example of the bottom reduced pipe dream.

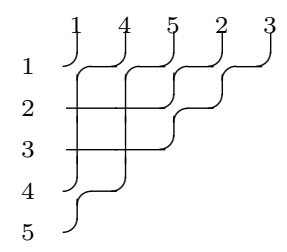

Fig. 5: The bottom reduced pipe dream for [14523] obtained by aligning the diagram to the left.

Consider the bottom reduced pipe dream drawn inside $R(\pi)$ and with elbows replaced by dots. Drop these dots south. Define $T(\pi)$ to be the tree with vertices $V=\left\{\alpha_{1}, \ldots, \alpha_{m}\right\}$ such that there is an edge between vertices $\alpha_{i}$ and $\alpha_{j}$ with $i<j$ if there is a dot in the entry in the column of the E step labeled $\alpha_{i} i$ and in the row of the $\mathrm{N}$ step labeled $\alpha_{i} j$. See Figure 6 for an example.
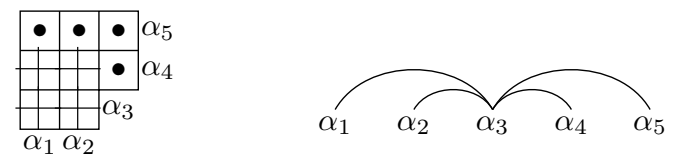

Fig. 6: On the left we have $N W(\pi)-\operatorname{Ess}(\pi)$ for $\pi=[14523]$ with its SW boundary labelled by $\left(\alpha_{1}, \ldots, \alpha_{m}\right)$ and the bottom reduced pipe dream drawn inside $N W(\pi)-E s s(\pi)$ with dots instead of elbows. We then drop the dots to the south to get the edges of $T(\pi)$, which is depicted on the right.

Theorem 4.4 Let $\pi=1 \pi^{\prime} \in S_{n}$, where $\pi^{\prime}$ is dominant. Let $\mathcal{C}^{2}(\pi)$ be the core of $P D(\pi)$ coned over twice. The canonical triangulation of the root polytope $\mathcal{P}(T(\pi))$ is a geometric realization of $\mathcal{C}^{2}(\pi)$.

We now mention some of the corollaries to this Theorem.

Corollary 4.5 The volume of the root polytope $\mathcal{P}\left(T\left(1 \pi^{\prime}\right)\right)$, for $\pi^{\prime}$ dominant is equal to the number of reduced pipe dreams of $1 \pi^{\prime}$. 
Recall that for a polytope $\mathcal{P} \subset \mathbb{R}^{N}$, the $t^{t h}$ dilate of $\mathcal{P}$ is $t \mathcal{P}=\left\{\left(t x_{1}, \ldots, t x_{N}\right) \mid\left(x_{1}, \ldots, x_{N}\right) \in \mathcal{P}\right\}$. The number of lattice points of $t \mathcal{P}$, where $t$ is a nonnegative integer and $\mathcal{P}$ is a convex polytope, is given by the Ehrhart function $i(\mathcal{P}, t)$. If $\mathcal{P}$ has integral vertices then $i(\mathcal{P}, t)$ is a polynomial.

In the spirit of Knutson and Miller (2004); Fomin and Kirillov (1994), the double $\beta$-Grothendieck polynomial $\mathfrak{G}_{w}^{\beta}(\mathbf{x}, \mathbf{y})$ for $w \in S_{n}$, where $\mathbf{x}=\left(x_{1}, \ldots, x_{n-1}\right)$ and $\mathbf{y}=\left(y_{1}, \ldots, y_{n-1}\right)$ is

$$
\mathfrak{G}_{w}^{\beta}(\mathbf{x}, \mathbf{y})=\sum_{P \in \operatorname{Pipes}(w)} \beta^{\operatorname{codim}_{P D(w)} F(P)} w t_{x, y}(P),
$$

where $\operatorname{Pipes}(w)$ is the set of all pipe dreams of $w$ (both reduced and nonreduced), $F(P)$ is the interior face in $P D(w)$ labeled by the pipe dream $P$, $\operatorname{codim}_{P D(w)} F(P)$ denotes the codimension of $F(P)$ in $P D(w)$ and $w t_{x, y}(P)=\prod_{(i, j) \in \operatorname{cross}(P)}\left(x_{i}-y_{j}\right)$, with $\operatorname{cross}(P)$ being the set of positions where $P$ has a cross. Note that in the product $\prod_{(i, j) \in \operatorname{cross}(P)}\left(x_{i}-y_{j}\right)$ we are assuming a certain labeling of rows and columns. Conventionally, rows are labeled increasingly from top to bottom and columns are labeled increasingly from left to right.

Corollary 4.6 Let $\pi=1 \pi^{\prime}$, where $\pi^{\prime}$ is a dominant permutation. Then

$$
\mathfrak{G}_{\pi}^{\beta-1}(\mathbf{1}, \mathbf{0})=\sum_{m \geq 0}\left(i(\mathcal{P}(T(\pi)), m) \beta^{m}\right)(1-\beta)^{\operatorname{dim}(\mathcal{P}(T(\pi)))+1} .
$$

\section{Degeneration of moment polytopes into acyclic root polytopes}

In this section we explain how to map the root polytope $\Phi\left(\mathbb{P}\left(Y_{\pi}\right)\right)$ to the acyclic root polytope $\mathcal{P}(T(\pi))$. We then use this map to triangulate $\Phi\left(\mathbb{P}\left(Y_{\pi}\right)\right)$ based on the triangulation of $\mathcal{P}(T(\pi))$.

Theorem 5.1 Given $\pi=1 \pi^{\prime}$, with $\pi^{\prime}$ dominant, the moment polytope $\Phi\left(\mathbb{P}\left(Y_{\pi}\right)\right)$ can be degenerated into the root polytope $\mathcal{P}(T(\pi))$.

Proof: Consider the linear map from $\Phi\left(\mathbb{P}\left(Y_{\pi}\right)\right) \rightarrow \mathcal{P}(T(\pi))$ that is the composition of the maps $K$ and $L$, where $L$ is the map

$$
\begin{aligned}
& L\left(x_{i}\right)=-e_{j}, \text { where } \alpha_{j} \text { is the label of step } N \text { on row } i, \text { and } \\
& L\left(y_{i}\right)= \begin{cases}0 & \text { if }(a, i) \in \operatorname{Ess}(\pi) \text { for some } a, \\
-e_{j} & \text { where } \alpha_{j} \text { is the label of step } E \text { on column } i\end{cases}
\end{aligned}
$$

and $K$ is the map given by

$$
\begin{aligned}
& K\left(y_{j}\right)= \begin{cases}x_{i} & \text { if }(i, j) \in \operatorname{Ess}(\pi), \\
y_{j} & \text { if there is no } a \text { such that }(a, j) \in \operatorname{Ess}(\pi) .\end{cases} \\
& K\left(x_{i}\right)=x_{i} .
\end{aligned}
$$

See Figure 8 for an example of these maps. Then this maps $\Phi\left(\mathbb{P}\left(Y_{\pi}\right)\right)$ to $\mathcal{P}(T(\pi))$.

The degeneration $L \circ K: \Phi\left(\mathbb{P}\left(Y_{\pi}\right)\right) \rightarrow \mathcal{P}(T(\pi))$ consists of contracting the face of $\Phi\left(\mathbb{P}\left(Y_{\pi}\right)\right)$ corresponding to $E s s(\pi)$ to a point and moving this point to the origin while tweaking the vertices of $\Phi\left(\mathbb{P}\left(Y_{\pi}\right)\right)$ that are of the form $\frac{1}{2}\left(x_{i}-y_{j}\right)$ where $(i, j)$ is north of an entry of $\operatorname{Ess}(\pi)$ and not in $\operatorname{dom}(\pi)$. 


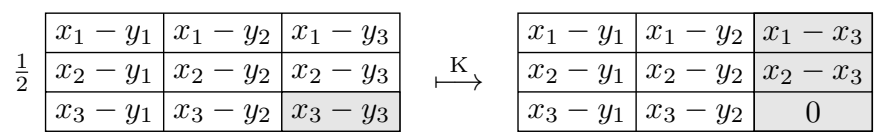

Fig. 7: The map $K$ for $Y_{[1243]}$.

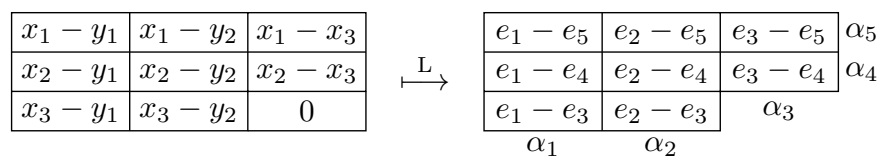

Fig. 8: The map $L$ for $Y_{[1243]}$.

\subsection{Triangulating $\Phi\left(\mathbb{P}\left(Y_{\pi}\right)\right)$ and geometric realization of subword complexes}

The preimage of the canonical triangulation of $\mathcal{P}(T(\pi))$ for $\pi=1 \pi^{\prime}$, with $\pi^{\prime}$ dominant, under the linear map $L \circ K$ is a triangulation of $\Phi\left(\mathbb{P}\left(Y_{\pi}\right)\right)$. This is yet another way to geometrically realize the pipe dream complex $P D(\pi)$ for these permutations.

Theorem 5.2 Let $\Delta_{1}, \ldots, \Delta_{k}$ be the top dimensional simplices in the canonical triangulation of $\mathcal{P}(T(\pi))$ for $\pi=1 \pi^{\prime}$, where $\pi^{\prime}$ is dominant. Then $P_{i}:=(L \circ K)^{-1}\left(\Delta_{i}\right), i \in[k]$, are the top dimensional simplices in a triangulation of $\Phi\left(\mathbb{P}\left(Y_{\pi}\right)\right)$ which we call its canonical triangulation.

Denote by $\mathcal{C}(\pi)$ the core of the pipe dream complex $P D(\pi)$ and by $\mathcal{C}^{i}(\pi)$ the core $\mathcal{C}(\pi)$ coned over $i$ times.

Theorem 5.3 The canonical triangulation of $\Phi\left(\mathbb{P}\left(Y_{\pi}\right)\right)$, for $\pi=1 \pi^{\prime}$, with $\pi^{\prime}$ dominant, is a geometric realization of $\mathcal{C}^{|\operatorname{Ess}(\pi)|+1}(\pi)$. Using the characterization of toric $Y_{\pi}$ of Theorem 3.3 , one can extend this geometric realization to realizations of pipe dream complexes for all $\pi$ such that $Y_{\pi}$ is toric.

\section{Acknowledgements}

We are grateful to Allen Knutson for many valuable discussions. We thank Maksim Maydanskiy for his interest and indepth comments on our research. We also thank Vic Reiner and Ed Swartz for several interesting and helpful conversations. Special thanks also go to Sergey Fomin for an extensive conversation about Grothendieck polynomials.

\section{References}

N. Bergeron and S. Billey. RC-graphs and Schubert polynomials. Experiment. Math., 2(4):257-269, 1993. ISSN 1058-6458. URL http://projecteuclid.org/euclid.em/1048516036.

N. Bergeron, C. Ceballos, and J.-P. Labbé. Fan realizations of type $A$ subword complexes and multiassociahedra of rank 3. Discrete Comput. Geom., 54(1):195-231, 2015. ISSN 0179-5376. doi: 10. 1007/s00454-015-9691-0. URL http://dx.doi.org/10.1007/s00454-015-9691-0. 
N. Berline and M. Vergne. Hamiltonian manifolds and moment map. 2011. http://www. math. polytechnique.fr/ berline/cours-Fudan.pdf.

A. Cannas da Silva. Lectures on symplectic geometry, volume 1764 of Lecture Notes in Mathematics. Springer-Verlag, Berlin, 2001. ISBN 3-540-42195-5. doi: 10.1007/978-3-540-45330-7. URL http: //dx.doi.org/10.1007/978-3-540-45330-7.

A. Cannas da Silva. Symplectic toric manifolds. In Symplectic geometry of integrable Hamiltonian systems (Barcelona, 2001), Adv. Courses Math. CRM Barcelona, pages 85-173. Birkhäuser, Basel, 2003.

C. Ceballos. On associahedra and related topics. PhD thesis, Freie Universität Berlin, Berlin, 2012.

C. Ceballos, J.-P. Labbé, and C. Stump. Subword complexes, cluster complexes, and generalized multi-associahedra. J. Algebraic Combin., 39(1):17-51, 2014. ISSN 0925-9899. doi: 10.1007/ s10801-013-0437-x. URL http://dx.doi.org/10.1007/s10801-013-0437-x.

P. Cellini and M. Marietti. Root polytopes and Abelian ideals. J. Algebraic Combin., 39(3):607-645, 2014. ISSN 0925-9899. doi: 10.1007/s10801-013-0458-5. URL http://dx.doi.org/10 . $1007 / \mathrm{s} 10801-013-0458-5$.

C. De Concini and V. Lakshmibai. Arithmetic Cohen-Macaulayness and arithmetic normality for Schubert varieties. Amer. J. Math., 103(5):835-850, 1981. ISSN 0002-9327. doi: 10.2307/2374249. URL http://dx.doi.org/10.2307/2374249.

L. Escobar and K. Mészáros. Subword complexes via triangulations of root polytopes. 2015a. arXiv:1502.03997.

L. Escobar and K. Mészáros. Toric matrix schubert varieties and their polytopes. 2015b. arXiv:1508.03445.

S. Fomin and A. N. Kirillov. Grothendieck polynomials and the Yang-Baxter equation. In Formal power series and algebraic combinatorics/Séries formelles et combinatoire algébrique, pages 183-189. DIMACS, Piscataway, NJ, 1994.

W. Fulton. Flags, Schubert polynomials, degeneracy loci, and determinantal formulas. Duke Math. J., 65(3):381-420, 1992. ISSN 0012-7094. doi: 10.1215/S0012-7094-92-06516-1. URL http: //dx.doi.org/10.1215/S0012-7094-92-06516-1.

I. M. Gelfand, M. I. Graev, and A. Postnikov. Combinatorics of hypergeometric functions associated with positive roots. In The Arnold-Gelfand mathematical seminars, pages 205-221. Birkhäuser Boston, Boston, MA, 1997. doi: 10.1007/978-1-4612-4122-5_10. URL http://dx.doi.org/10.1007/ 978-1-4612-4122-5_10.

A. Knutson and E. Miller. Subword complexes in Coxeter groups. Adv. Math., 184(1):161-176, 2004. ISSN 0001-8708. doi: 10.1016/S0001-8708(03)00142-7. URL http://dx.doi.org/10.1016/ S0001-8708(03)00142-7 
A. Knutson and E. Miller. Gröbner geometry of Schubert polynomials. Ann. of Math. (2), 161(3):12451318, 2005. ISSN 0003-486X. doi: 10.4007/annals.2005.161.1245. URL http://dx.doi.org/ $10.4007 / a n n a l s .2005 .161 .1245$

K. Mészáros. Root polytopes, triangulations, and the subdivision algebra. I. Trans. Amer. Math. Soc., 363(8):4359-4382, 2011. ISSN 0002-9947. doi: 10.1090/S0002-9947-2011-05265-7. URL http: //dx.doi.org/10.1090/S0002-9947-2011-05265-7.

K. Mészáros. h-polynomials of reduction trees. 2014. arXiv:1407.2684.

K. Mészáros. Calculating Greene's function via root polytopes and subdivision algebras. 2015. arXiv:1508.01301.

K. Mészáros. $h$-polynomials via reduced forms. Electron. J. Combin., 22(4):Paper 4.18, 17, 2015. ISSN 1077-8926.

K. Mészáros. Product formulas for volumes of flow polytopes. Proc. Amer. Math. Soc., 143(3):937-954, 2015. ISSN 0002-9939. doi: 10.1090/S0002-9939-2014-12182-4. URL http://dx.doi.org/ $10.1090 / 50002-9939-2014-12182-4$

K. Mészáros. Pipe Dream Complexes and Triangulations of Root Polytopes Belong Together. SIAM J. Discrete Math., 30(1):100-111, 2016. ISSN 0895-4801. doi: 10.1137/15M1014802. URL http: //dx.doi.org/10.1137/15M1014802.

K. Mészáros and A. H. Morales. Flow polytopes of signed graphs and the Kostant partition function. International Mathematical Research Notices, (3):830-871, 2015.

V. Pilaud and M. Pocchiola. Multitriangulations, pseudotriangulations and primitive sorting networks. Discrete Comput. Geom., 48(1):142-191, 2012. ISSN 0179-5376. doi: 10.1007/s00454-012-9408-6. URL http://dx.doi.org/10.1007/s00454-012-9408-6.

V. Pilaud and F. Santos. The brick polytope of a sorting network. European J. Combin., 33(4):632-662, 2012. ISSN 0195-6698. doi: 10.1016/j.ejc.2011.12.003. URL http://dx.doi.org/10.1016/ j.ejc.2011.12.003.

S. Ramanan and A. Ramanathan. Projective normality of flag varieties and Schubert varieties. Invent. Math., 79(2):217-224, 1985. ISSN 0020-9910. doi: 10.1007/BF01388970. URL http://dx.doi. org/10.1007/BF01388970

L. Serrano and C. Stump. Maximal fillings of moon polyominoes, simplicial complexes, and Schubert polynomials. Electron. J. Combin., 19(1):Paper 16, 18, 2012. ISSN 1077-8926.

C. Stump. A new perspective on $k$-triangulations. J. Combin. Theory Ser. A, 118(6):1794-1800, 2011. ISSN 0097-3165. doi: 10.1016/j.jcta.2011.03.001. URL http://dx.doi.org/10.1016/j. jeta.2011.03.001. 